\title{
A Norm Utilisation for Scarce Hospital Resources: Evidence from Operating Rooms in a Dutch University Hospital
}

\author{
Mark Van Houdenhoven • Erwin W. Hans • Jan Klein • \\ Gerhard Wullink • Geert Kazemier
}

Received: 14 November 2006 / Accepted: 28 February 2007 / Published online: 19 April 2007

(C) Springer Science + Business Media, LLC 2007

\begin{abstract}
Background Utilisation of operating rooms is high on the agenda of hospital managers and researchers. Many efforts in the area of maximising the utilisation have been focussed on finding the holy grail of $100 \%$ utilisation. The utilisation that can be realised, however, depends on the patient mix and the willingness to accept the risk of working in overtime.

Materials and methods This is a mathematical modelling study that investigates the association between the utilisation and the patient mix that is served and the risk of
\end{abstract}

M. Van Houdenhoven · G. Wullink · G. Kazemier

Division of Operating Rooms, ICU, and Anaesthesiology, ErasmusMC University Medical Centre,

Rotterdam, The Netherlands

\section{E. W. Hans}

Department of Operational Methods for Production and Logistics, School of Management and Governance, University of Twente, Enschede, The Netherlands

\section{J. Klein}

Department of Anaesthesiology,

ErasmusMC University Medical Centre,

Rotterdam, The Netherlands

G. Wullink $(\bowtie)$

Division of Operating Rooms, ICU, and Anaesthesiology, ErasmusMC University Medical Center, Dr. Molewaterplein 40, 3015 GD, Rotterdam, The Netherlands

e-mail: g.wullink@erasmusmc.nl

\section{G. Kazemier}

Department of Surgery,

ErasmusMC University Medical Centre,

Rotterdam, The Netherlands working in overtime. Prospectively, consecutively, and routinely collected data of an operating room department in a Dutch university hospital are used. Basic statistical principles are used to establish the relation between realistic utilisation rates, patient mixes, and accepted risk of overtime.

Results Accepting a low risk of overtime combined with a complex patient mix results a low utilisation rate. If the accepted risk of overtime is higher and the patient mix is less complex, the utilisation rate that can be reached is closer to $100 \%$.

Conclusion Because of the inherent variability of healthcare processes, the holy grail of $100 \%$ utilisation is unlikely to be found. The method proposed in this paper calculates a realistic benchmark utilisation that incorporates the patient mix characteristics and the willingness to accept risk of overtime.

Keywords Hospitals $\cdot$ Health care costs $\cdot$ Econometrics

\section{Introduction}

The utilisation rate is commonly used as an indicator to measure the efficiency of the use of hospital resources such as the operating rooms (OR). Researchers often argue that high utilisation is cost-effectiveness and goes hand in hand with high quality of health care [1-6]. In these studies utilisation is typically considered as an absolute measure that can be used to resolve the perceived efficiency problem in health care [7-11]. In accordance with this trend, governmental institutions, such as the audit commission of the National Health-care Service, offer programmes to improve utilisation of operating room departments 
Fig. 1 Representation of the measurement moment at a single operating room. The ratio between the Occupied time and the Available block time defines the utilisation of the operating room. Anaesthetic time, cleaning time, and changeover times are included in the case durations

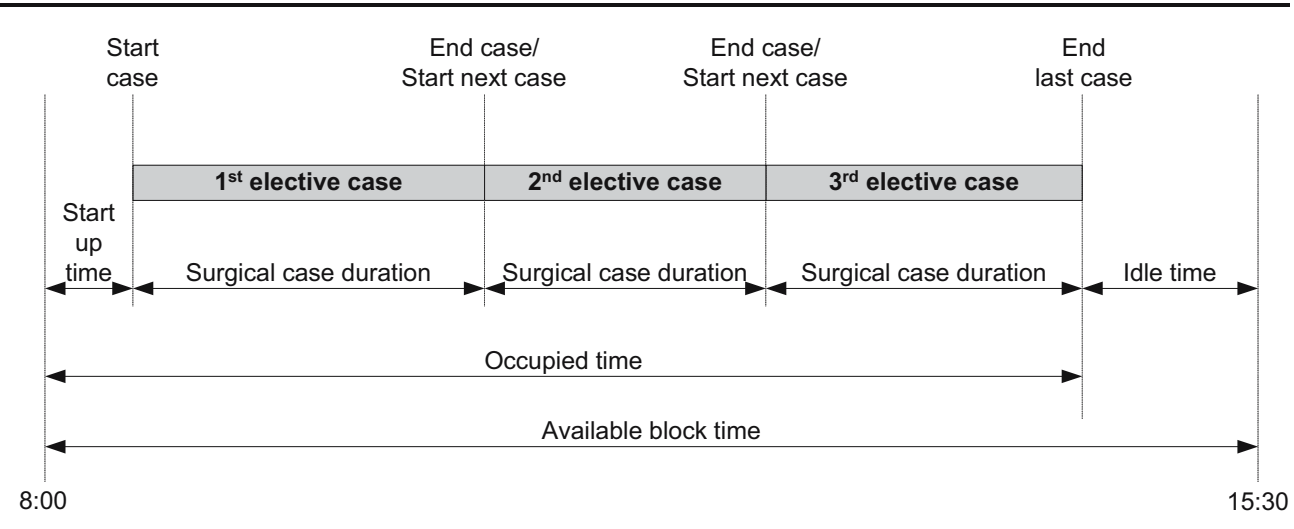

$[12,13]$. Practice learns, however, that utilisation rates of $100 \%$ are rarely achieved.

While trying to maximise the utilisation of the OR, managers are confronted with the complexity of the case mix and high costs for overtime of the OR staff. Typically, in hospitals with a complex case mix with a high variability in the duration of surgical cases, utilisation rates are significantly lower than $100 \%$. For example, $100 \%$ utilisation for a cancer medical centre is utopian, since a considerable number of the surgical cases cannot be completed within the planned duration because of the unpredictability of the disease. Typically in a cancer centre the duration of surgical cases is unpredictable and they can last much longer than expected. This causes frequent overtime in operating rooms of a cancer centre.

The aim of this study was to address the association between operating room utilisation, the case mix, and the accepted risk of overtime, using straightforward statistical analyses. For this purpose, we propose a method to establish this association by calculating a norm utilisation for the OR. We compared the sensitivity of the method with respect to the two main input parameters, namely the case mix and the accepted risk of overtime. The study uses prospectively collected data of the Erasmus Medical Centre (Erasmus MC) in Rotterdam, The Netherlands.

\section{Materials and methods}

The records of 180,000 surgical cases performed at Erasmus MC's main location from January 1, 1994 through December 31, 2005, were used. The following surgical departments have been investigated: General Surgery, Gynaecology, Oral Surgery, Ear-Nose-Throat Surgery, Neurosurgery, Ophthalmology, Orthopaedic Surgery, Plastic Surgery, and Urologic Surgery. All data were entered electronically by the nursing staff in the Hospital Information System and validated by the responsible surgeon and anaesthetist. Based on this extensive database we computed several case mix characteristics such as the standard deviation of the duration of elective and emergency cases and the number of elective and emergency cases per specialty. For this study, we assumed that the number of cases performed, the average duration, and the standard deviation for both elective and emergency cases describe
Fig. 2 A schematic representation of a surgical case plan of an operating room. Reserved capacity is included to perform emergency surgical cases and to deal with the variability of the overall completion time

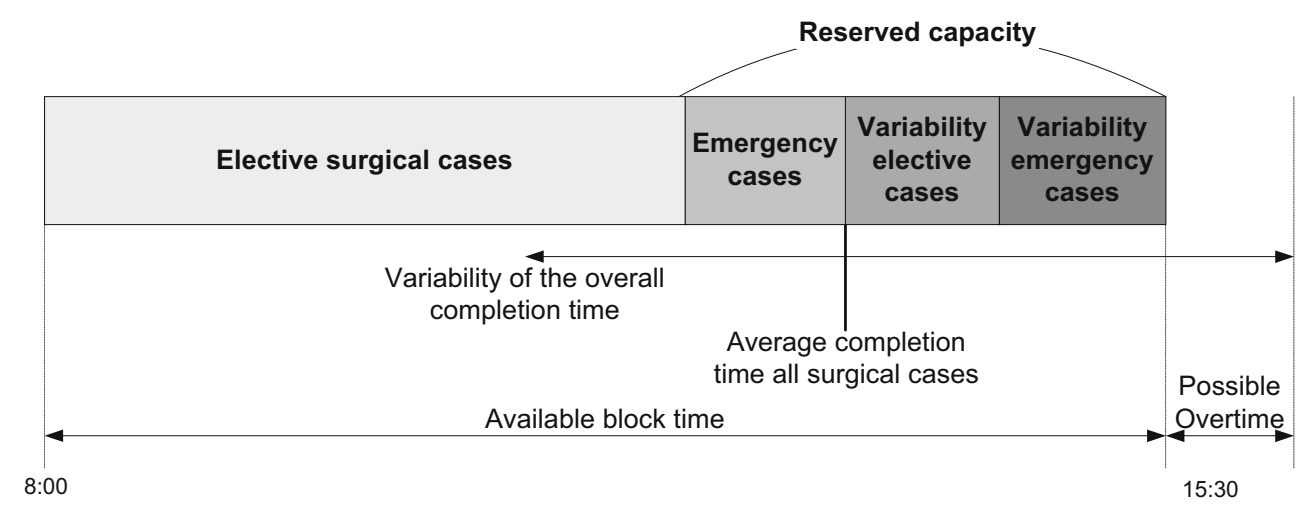


Table 1 Overview of mathematical notation used in the research

\begin{tabular}{|c|c|}
\hline Symbol & Meaning \\
\hline$s$ & A surgical department \\
\hline$\mu_{s}$ & Average elective case duration of surgical department $s$ \\
\hline$\sigma_{s}$ & $\begin{array}{l}\text { Standard deviation of the duration of elective cases of } \\
\text { surgical department } s\end{array}$ \\
\hline$n_{s}$ & $\begin{array}{l}\text { Average number of elective cases per OR block of surgical } \\
\text { department } s\end{array}$ \\
\hline$\mu_{s}^{e}$ & Average emergency case duration of surgical department $s$ \\
\hline$\sigma_{s}^{e}$ & $\begin{array}{l}\text { Standard deviation of the duration of emergency cases of } \\
\text { surgical department } s\end{array}$ \\
\hline$n_{s}^{e}$ & $\begin{array}{l}\text { Average number of emergency cases per OR block of } \\
\text { surgical department } s\end{array}$ \\
\hline$r_{s}$ & Accepted risk of overtime for surgical department $s$ \\
\hline$\mu$ & Overall average elective case duration \\
\hline$\mu^{e}$ & Overall average emergency case duration \\
\hline$\sigma$ & Overall standard deviation of elective cases \\
\hline$\sigma^{e}$ & Overall standard deviation of emergency cases \\
\hline
\end{tabular}

the patient mix. Figure 1 depicts all measurement moments that were used in this study.

We assumed that cases were scheduled using the block planning approach [14]. In this case scheduling approach, a surgical department schedules its elective surgical cases in the blocks of operating time that are available for that surgical department. Scheduling surgical cases is done using average case durations. To reduce the risk of overtime induced by emergency cases and the variability of the duration of elective and emergency cases the surgical department has to plan reserve capacity in the OR block $[15,16]$. The amount of reserve capacity is calculated on basis of the variability of the duration of elective and emergency surgical cases.

Reserve capacity to operate upon emergency surgical cases is assigned to all elective operating rooms. Hence, no emergency operating room exists. Upon arrival the decision is made in which operating room the emergency case will take place, the emergency patient has to wait until the preceding cases in this operating room is ended. The total amount of reserved capacity for emergency cases is based on the average duration and the expected number of emergency surgical cases. Hence, the reserved OR capacity is used to deal with the variability of elective cases, emergency cases, and to deal with the variability of emergency cases. Figure 2 shows this schematically.

There are various ways to compute the utilisation rate. In this study the utilisation of OR capacity is defined as the time an operating room is occupied to perform elective and emergency surgical cases, expressed as a percentage of the length of time an operating room is available and staffed during a certain period (see Fig. 1) [14, 17].

The method requires some mathematical notation (see Table 1). Let $s$ denotes a surgical department. The average number of elective surgical cases executed in an OR block by surgical department $s$ is $n_{s} . \mu_{s}$ is the average duration and $\sigma_{s}$ the standard deviation of a surgical case of department $s$. Emergency surgery for surgical department $s$ is characterised by the average duration of the emergency surgical case $\left(\mu_{s}^{e}\right)$, the standard deviation of the duration $\left(\sigma_{s}^{e}\right)$, and the average number of emergency cases per OR block $\left(n_{s}^{e}\right)$. The accepted risk of overtime for surgical department $s$ is denoted by $r_{s}$.

The following steps were taken to calculate the amount of OR time that is required for operating all elective and emergency cases given the variability and a predetermined accepted risk of overtime. The total expected duration of all elective cases in one OR block is $n_{s} \cdot \mu_{s}$. The standard deviation of the total duration of these $n_{s}$ cases equals $\sqrt{n_{s} \cdot \sigma_{s}^{2}}$. The total capacity to operate all elective cases of surgical department $s$ is thus $n_{s} \cdot \sigma_{s}+\alpha\left(r_{s}\right) \sqrt{n_{s} \cdot \sigma_{s}^{2}}$, where $\alpha\left(r_{s}\right)$ is a function that gives a factor that yields a probability $r_{s}$ of working in overtime. The outcome of this function depends on the distribution of the total duration of $n_{s}$ cases. For simplicity we shall assume here that this is a normal distribution. In this case, suppose $r_{s}=50 \%$, then it follows from a normal distribution that $\alpha\left(r_{s}\right)=0$. The

Table 2 Input values based on data of Erasmus MC

\begin{tabular}{|c|c|c|c|c|c|c|c|c|c|c|}
\hline & SUR & GYN & ORAL & ENT & NEC & TRAU & OPT & ORT & PLC & URO \\
\hline Total available time per day & 1,350 & 450 & 450 & 450 & 450 & 450 & 450 & 450 & 900 & 450 \\
\hline Average number of cases per day $n_{s}$ & 6.8 & 3.7 & 2.5 & 2.7 & 1.3 & 3.2 & 6.0 & 2.6 & 4.8 & 2.9 \\
\hline Average case duration (minutes) $\mu_{s}$ & 172 & 104 & 150 & 119 & 256 & 108 & 67 & 136 & 154 & 125 \\
\hline Average case standard deviation (minutes) $\sigma_{s}$ & 106 & 56 & 88 & 135 & 157 & 60 & 34 & 77 & 133 & 92 \\
\hline Number emergency surgical cases per day $\left(n_{s}^{e}\right)$ & 0.9 & 0.1 & 0.0 & 0.1 & 0.2 & 0.3 & 0.0 & 0.2 & 0.2 & 0.1 \\
\hline Average emergency case duration (minutes) $\left(\mu_{s}^{e}\right)$ & 132 & 76 & 134 & 87 & 141 & 138 & 107 & 118 & 174 & 93 \\
\hline $\begin{array}{l}\text { Average emergency standard deviation (minutes) } \\
\left(\sigma_{s}^{e}\right)\end{array}$ & 103 & 47 & 75 & 69 & 87 & 89 & 62 & 75 & 166 & 51 \\
\hline
\end{tabular}


Table 3 Results, Erasmus MC with an accepted risk of overtime of 31\%

\begin{tabular}{|c|c|c|c|c|c|c|c|c|c|c|}
\hline & SUR & GYN & ORAL & ENT & NEC & TRAU & OPT & ORT & PLC & URO \\
\hline Reserved capacity per OR block (minutes) & 147 & 55 & 70 & 112 & 92 & 59 & 43 & 65 & 150 & 78 \\
\hline Norm utilisation (\%) & 90 & 88 & 84 & 75 & 80 & 87 & 91 & 86 & 84 & 83 \\
\hline
\end{tabular}

chosen total capacity would thus be $n_{s} \cdot \mu_{s}$. Or, suppose $r_{s}=$ $31 \%$, then $\alpha\left(r_{s}\right)=0.5$, and the chosen total capacity would be $n_{s} \cdot \mu_{s}+0.5 \sqrt{n_{s} \cdot \sigma_{s}^{2}}$. The term $\alpha\left(r_{s}\right) \sqrt{n_{s} \cdot \sigma_{s}^{2}}$ is thus the total required reserve capacity. This approach does not depend on the chosen distribution; if another distribution is used (e.g. the sum of lognormal distributions), only the outcome of $\alpha\left(r_{s}\right)$ changes. The capacity to execute the expected number of emergency cases is determined analogously: $n_{s}^{e} \cdot \mu_{s}^{e}+\alpha\left(r_{s}\right) \sqrt{n_{s}^{e} \cdot\left(\sigma_{s}^{e}\right)^{2}}$. If elective and emergency cases are scheduled together in one OR, the total reserve capacity that guarantees an overtime risk $r_{s}$ for surgical department $s$ is: $\alpha\left(r_{s}\right) \cdot \sqrt{n_{s} \cdot\left(\sigma_{s}\right)^{2}+n_{s}^{e} \cdot\left(\sigma_{s}^{e}\right)^{2}}$. The utilisation rate for specialty $s$ is then:

$$
\frac{n_{s} \cdot \mu_{s}+n_{s}^{e} \cdot \mu_{s}^{e}}{n_{s} \cdot \mu_{s}+n_{s}^{e} \cdot \mu_{s}^{e}+\alpha\left(r_{s}\right) \cdot \sqrt{n_{s} \cdot\left(\sigma_{s}\right)^{2}+n_{s}^{e} \cdot\left(\sigma_{s}^{e}\right)^{2}}}
$$

To investigate the sensitivity of the outcome of the formula we have varied the standard deviation of the case duration to evaluate the effect of different case mixes on utilisation rates. We also investigated the sensitivity of the utilisation with respect to various values of $r$, i.e., the predetermined accepted risk of overtime.

\section{Results}

Table 2 summarises the Erasmus MC data, which has been the input of all experiments. The accepted risk of overtime in Erasmus MC is $31 \%$.
Applied to the case of General Surgery in the Erasmus MC the proposed formulas result in the following computations:

- The time required to perform elective and emergency cases: $n_{s} \cdot \mu_{s}+n_{s}^{e} \cdot \mu_{s}^{e}=6.77 \cdot 172.39+0.94 \cdot 131.75=$ $1,167 \mathrm{~min}$;

- The factor for calculating the required reserved capacity is $\alpha(31 \%)=0.5$;

- Required reserved capacity: $\alpha\left(r_{s}\right) \cdot \sqrt{n_{s} \cdot\left(\sigma_{s}\right)^{2}+n_{s}^{e} \cdot\left(\sigma_{s}^{e}\right)^{2}}=$ $\alpha(31 \%) \cdot \sqrt{6.77 \cdot(106.32)^{2}+0.94 \cdot(102.78)^{2}}=147 \mathrm{~min} ;$

- Utilisation of operating room time: $1,167 /(1,167+147)$. $100 \%=90 \%$

Table 3 shows that, given an accepted risk of overtime of $31 \%$, the utilisation varies between $75 \%$ (for Ear, Nose, and Throat surgery, including head and neck surgery) and $91 \%$ (for Ophthalmology).

The association between the patient mix, represented by the variability of the case durations, and utilisation and the association between th e accepted risk of overtime and utilisation is shown for three surgical departments: General Surgery, Ear-Nose-Throat Surgery, and Ophthalmology. Figure 3 addresses the correlation between the variability in the duration of cases and utilisation. As may be expected, if there is no variability in the duration of the surgery, the utilisation is $100 \%$. Utilisation decreases, however, as variability increases, since the required reserved capacity depends on the variability. Utilisation increases from 89 to $95 \%$ if the standard deviation is varied from the standard deviation of General Surgery (Erasmus MC) to half of this
Fig. 3 Utilisation versus variability in the standard deviation of the case duration

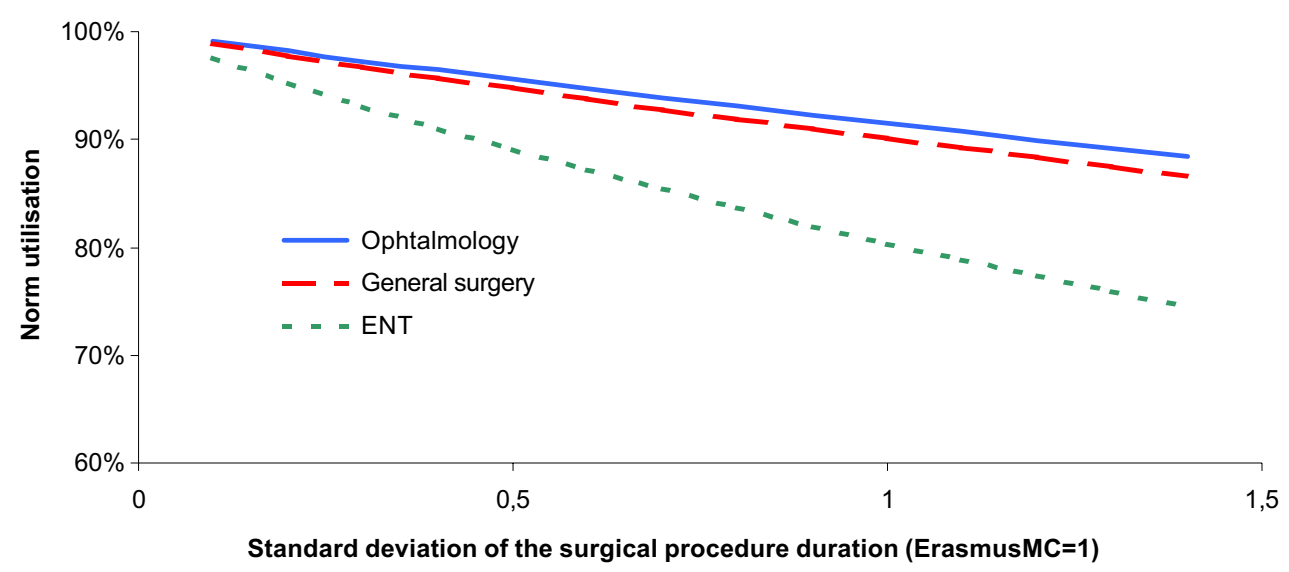


Fig. 4 Utilisation versus the accepted risk of overtime

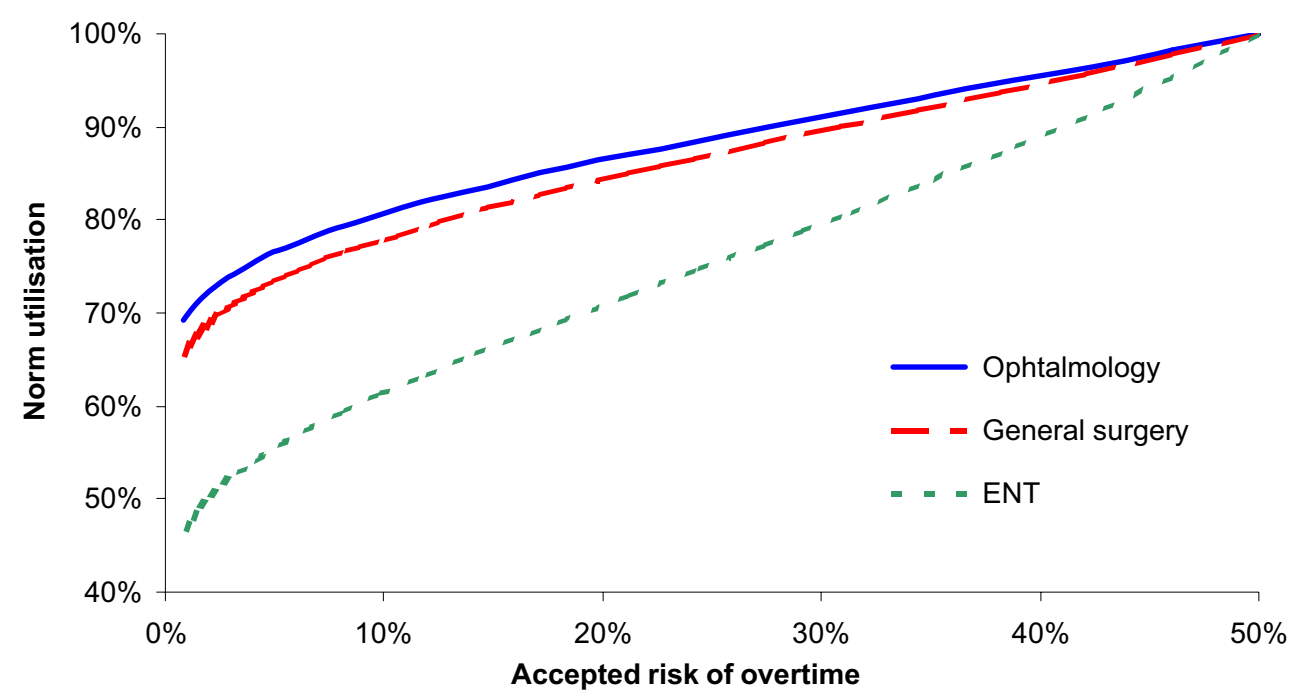

value. Figure 4 shows that the utilisation increases when the accepted risk of overtime grows.

\section{Discussion}

The relation between the utilisation of operating room time, patient mix, and the accepted risk of overtime was addressed. With relatively straightforward statistical techniques, the association between the required reserved capacity and the acceptable risk of overtime and the variability of the case mix are established. This results in a method to determine a realistic norm utilisation rate for a scarce hospital resource such as an operating room considering the complexity of the case mix and managerial choices such as working in overtime.

Little research has been performed to the association between an operating room patient mix, willingness to accept risk of overtime, and operating room performance in terms of capacity utilisation. Some work on utilisation rates acknowledges the limitations of utilisation as a performance measure [18]. Strum et al. focus on cost efficiency of the OR and use a minimal cost analysis model to make the explicit trade-off between the costs for over and underutilisation [19]. Their approach serves to determine the best OR time allocation to specialties on a daily basis. As far as we know, a generic method that establishes the association between patient mix characteristics and managerial choices, such as working in overtime, lacks. The calculations presented in this paper do not capture the entire complex reality of health care. The assumptions and simplifications, however, do not violate the general idea of the approach. For instance, following the central limit theorem of statistics, we assumed a normal distribution for the total duration of the surgical cases. A more accurate distribution (e.g., a distribution based on the sum of lognormal distributions) can be used if sufficient historical data are available. The idea remains applicable but the complexity of the calculations increase.

Furthermore, we assumed an identical average and standard deviation for the duration of all the cases of a given surgical department. This assumption leads to a slight overestimation of the required reserve capacity and hence to a conservative norm utilisation. We did not account for cancelled cases. If a hospital has information about the average number of cancelled cases however, this can be incorporated in the formula for the norm utilisation. Finally, the amount of emergency cases may differ significantly over the days of the week. All of these aspects can be dealt with, without harming the general idea of the approach. There are several definitions of utilisation, which may yield different results, but rely on the same basic assumptions. Another definition to compute utilisation may yield slightly other rates, but the idea of the method proposed in the paper remains the same. Also, the reserve capacity that is computed with the proposed method can be allocated to one or more operating rooms. The computations and the resulting norm utilisation, however, remains the same regardless the way the reserve capacity is allocated.

The method proposed in this paper was developed to determine a utilisation rate for each surgical department individually. OR management can also choose to determine a single utilisation rate for the total department by not distinguishing between surgical departments. Omitting the s index in the proposed formula and using the case of all departments together can achieve this.

The operating room of the Erasmus MC was used as an example. The proposed method, however, can be used for many other scarce hospital resources to obtain realistic 
utilisation rates, such as the radiology department, wards or the intensive care.

The utilisation rate computed in this paper can be perceived as the theoretical maximum benchmark utilisation rate. It can be realised only if one assumes that the operating room department is making use of optimal planning techniques, has adequate management, makes the best use of its personnel, and has adequate equipment available to it, which is in practice exceptionally. Contrarily, aiming at a utilisation rate higher than the calculated utilisation is practically impossible given a risk of working in overtime and the variability of the case mix.

In operating room benchmarking studies, utilisation rates are typically compared without considering patient mix characteristics and managerial choices. These widely used comparisons typically overlook the key factors that actually determine utilisation. We conclude, therefore, that the use of utilisation as an absolute measure is an unfair comparison of hospitals departments and probably leads to the wrong conclusions. The method developed for computing the maximum achievable norm utilisation should be the basis for a new approach to operating room and hospital benchmarking and internal performance measurements for hospital boards.

In the various health-care systems, hospital funding is based on the number of surgical cases actually performed. If norm utilisation is not incorporated in the price agreed upon, a hospital will probably lack the resources to finance the required reserve capacity required to deal with the variability of the case mix and managerial choices. To ensure cost-efficient health care given the complexity of different types of health care these factors should be used into negotiations between financers and providers of health care.

This paper showed that reserving capacity results in a lower utilisation rate. Hospital boards under pressure to increase their utilisation may hence decide to achieve higher targets by refusing complex and emergency care that involves a high variance. From a societal perspective, such an eventuality is highly undesirable. To prevent this calculating behaviour, hospitals should be judged on their utilisation with respect to their own norm utilisation.

We proposed a method to determine a norm utilisation rate the operating room. This method accounts for the complexity of health care processes and the differences between hospitals. The method proposed can be used for managerial issues to evaluate and steer their own perfor- mance, but also externally to support contract negotiations and benchmarking of hospitals.

\section{References}

1. Gordon, T., Paul, S., Lyles, A., and Fountain, J., Surgical unit time utilization review: Resource utilization and management implications. J. Med. Syst. 12(3):169-179, 1988.

2. OECD Health Data 2005. IRDES.

3. Young, A., The empty theatre. BMJ 300(6735):1288-1289, 1990.

4. Dowdall, J. M., The use of operating theatres in the Northern Ireland Health and Personal Social Services. Northern Ireland Audit Office: Belfast, 2003.

5. Audit Commission. Operating theatres, Review of national findings, 2003.

6. Bevan Report. The Management and utilisation of operating departments, 1989.

7. Overdyk, F. J., Harvey, S. C., Fishman, R. L., and Shippey, F., Successful strategies for improving operating room efficiency at academic institutions. Anesth. Analg. 86(4):896-906, 1998.

8. Dexter, F., Macario, A., Lubarsky, D. A., and Burns, D. D., Statistical method to evaluate management strategies to decrease variability in operating room utilization: application of linear statistical modeling and Monte Carlo simulation to operating room management. Anesthesiology 91(1):262-274, 1999.

9. Dexter, F., Macario, A., and Traub, R. D., Which algorithm for scheduling add-on elective cases maximizes operating room utilization? Use of bin packing algorithms and fuzzy constraints in operating room management. Anesthesiology 91(5):1491-1500, 1999.

10. Kuo, P. C., Schroeder, R. A., Mahaffey, S., and Bollinger, R. R., Optimization of operating room allocation using linear programming techniques. J. Am. Coll. Surg. 197(6):889-895, 2003.

11. Eaton, L., Trusts cancel $10 \%$ of operating theatre sessions. $B M J$ 324(7347):1174, 2002.

12. Agency, N. M., Theatre programme, step guide to improving operating theatre performance, 2003.

13. Healthcare, C. F., Inspecting informing improving, acute hospital portfolio review, day surgery, 2005.

14. Viapiano, J., and Ward, D. S., Operating room utilization: The need for data. Int. Anesthesiol. Clin. 38(4):127-140, 2000.

15. Gallivan, S., Utley, M., Treasure, T., and Valencia, O., Booked inpatient admissions and hospital capacity: Mathematical modelling study. BMJ 324(7332):280-282, 2002.

16. Hans, E. W., Wullink, G., Houdenhoven, M., and Kazemier, G., Robust surgery loading. Eur. J. Oper. Res., 2006 (in press).

17. Mazzei, W. J., Maximizing operating room utilization: A landmark study. Anesth. Analg. 89(1):1-2, 1999.

18. Dexter, F., Macario, A., Traub, R. D., and Lubarsky, D. A., Operating room utilization alone is not an accurate metric for the allocation of operating room block time to individual surgeons with low caseloads. Anesthesiology 98(5):1243-1249, 2003.

19. Strum, D. P., Vargas, L. G., and May, J. H., Surgical subspecialty block utilization and capacity planning: A minimal cost analysis model. Anesthesiology 90(4):1176-1185, 1999. 